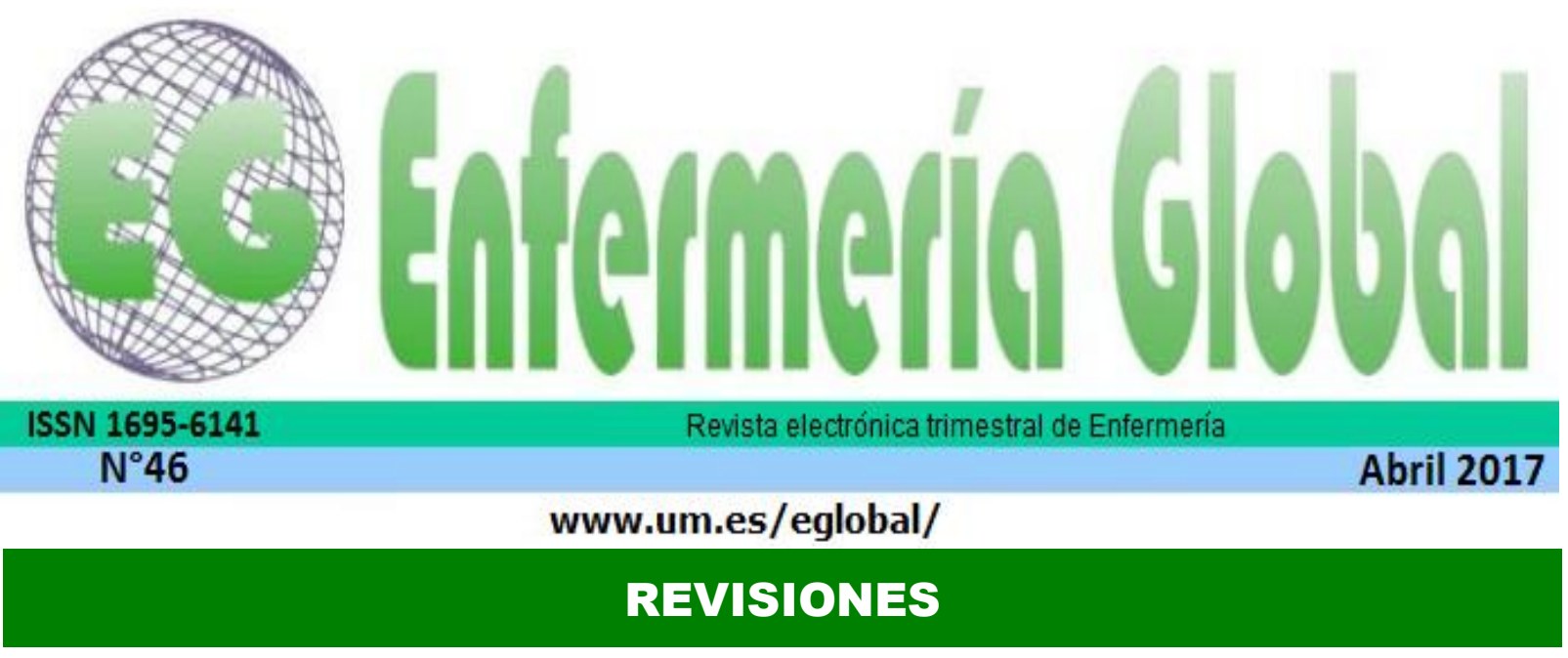

\title{
Revisión sistemática de las complicaciones de los dispositivos de administración de tratamiento al paciente oncológico
}

Systematic review of the complications of treatment delivery devices for cancer patients

\section{*Toril Rubio, Marina *Rodríguez Borrego, María Aurora}

*Departamento de Enfermería. Universidad de Córdoba. España. E-mail: marinatoril@hotmail.com

http://dx.doi.org/10.6018/eglobal.16.2.251571

\section{RESUMEN}

Objetivos: Conocer la producción científica que hay en relación a los dispositivos de administración de tratamiento al paciente oncológico, así como conocer las principales complicaciones de los Catéteres Venosos Centrales con reservorio (Port-A-Cath) y los Catéteres Centrales Insertados Percutáneamente (CCIP).

Metodología: Estudio de revisión sistemática de la literatura científica en las bases de datos, cuya recogida de datos se realizó de enero a mayo de 2015.

Resultados: Se seleccionaron un total de 124 artículos por título y resumen. De ellos, 42 documentos cumplieron los criterios de inclusión. La producción científica de los Port-A-Cath es mayor que de los CCIP y las complicaciones infecciosas y trombóticas son las más frecuentes en ambos dispositivos.

Conclusiones: La mayoría de las complicaciones pueden ser prevenidas ya que se desarrollan debido a errores en la implantación del dispositivo o a unos cuidados inapropiados. Es difícil determinar qué dispositivo proporciona mayor calidad de vida, aunque los pacientes suelen preferir los Port-A-Cath por su resultado estético.

Palabras clave: cáncer; Port-A-Cath; CCIP; quimioterapia; complicaciones

\section{ABSTRACT}

Objetives: To know the scientific production releated with the delivery treatment devices to the oncology patients and to know the main complications of central venous catheters with reservoir (PortA-Cath) and Percutaneously Inserted Central Catheters (PICC). 
Methods: Systematic review of the scientific literature in databases, whose data collection was realized from January to May 2015.

Results: 124 articles were selected by its title and abstract. 42 documents of them had the inclusion criteria. Port-A-Cath scientific production is greater than PICC's and infectious and thrombotic complications are the most common in both devices.

Conclusions: Most complications can be prevented because their development is due to errors in the implementation of the device or due to inappropriate care. It is difficult knowing which device provides better quality of life, although patients usually perfer Port-A-Cath because of its aesthetic result.

Keywords: cancer; Port-A-Cath; PICC; chemotherapy; complications.

\section{INTRODUCCIÓN}

La Sociedad Americana del Cáncer (American Cancer Society) define el cáncer como "el nombre general de un grupo de más de 100 enfermedades"(1), mientras que, por otro lado, el Instituto Nacional del Cáncer (National Cancer Institute) completa esta definición afirmando que "en todos los tipos de cáncer, algunas de las células del cuerpo empiezan a dividirse sin detenerse y se diseminan a los tejidos de alrededor"(2).

La Sociedad Española de Oncología Médica (SEOM) determinó que en 2012 hubo una prevalencia a 5 años de 581.688 casos de cáncer en España, independientemente de la fecha en la que fueron diagnosticados ${ }^{(3)}$. De este número de pacientes con cáncer, esta Sociedad ha registrado una mortalidad de 102.762 casos de fallecimientos ${ }^{(3)}$. Se puede afirmar que el cáncer es una enfermedad que ha ido ganando incidencia.

La quimioterapia intravenosa es uno de los tratamientos del cáncer más populares y tradicionales ${ }^{(1,4)}$, por lo que la implantación de un Catéter Venoso Central (CVC) a largo plazo es recomendable para los pacientes que requieren este tratamiento de larga duración. Dos de estos CVC son los dispositivos totalmente implantados con reservorio (Port-A-Cath) y los Catéteres Centrales Insertados Percutáneamente $(\mathrm{CCIP})^{(5)}$. A pesar de que ambos dispositivos presentan ventajas importantes para el paciente oncológico, también podrían suponer un riesgo debido a sus complicaciones. En base a esto surge la siguiente pregunta de investigación: ¿Cuáles son las complicaciones de los dispositivos con los que se puede administrar la quimioterapia intravenosa para el paciente oncológico?

Analizar la evidencia científica puede ayudar a esclarecer estas complicaciones y la forma de actuar ante ellas. Cortés-Flores AO et al. ${ }^{(6)}$ las clasifica como tempranas 0 tardías. Las complicaciones tardías son aquellas que ocurren después de los 30 primeros días de la inserción del dispositivo y emergen a largo plazo debido a una mala praxis. La frecuencia de complicaciones de Port-A-Cath se encuentra entre el 3$30 \%{ }^{(7)}$, mientras que la incidencia de complicaciones de los CCIP oscila entre el 5$26 \%{ }^{(8)}$.

En busca de respuesta se diseñó una revisión sistemática con el objetivo general de conocer la producción científica existente en relación con los dispositivos de administración de tratamiento al paciente oncológico, y con los objetivos específicos de conocer las principales complicaciones de la implantación y uso de los Port-A-Cath 
y los CCIP, establecer semejanzas y diferencias entre ambos accesos venosos, conocer el manejo de sus complicaciones y determinar cuál de ellos proporciona una mejor calidad de vida a los pacientes oncológicos.

\section{METODOLOGÍA}

Revisión sistemática de la literatura científica existente en las bases de datos PubMed, Google Académico, Health \& Medical Complete y SciELO, además del uso del buscador Google para la recogida de información en Sedes Webs.

Los criterios de inclusión para la selección de artículos científicos fueron las publicaciones dirigidas al estudio de Port-A-Cath y CCIP, en español e inglés, nacionales e internacionales y publicadas desde el año 2002 al 2015 . Se excluyeron aquellas publicaciones relacionadas con una patología concreta.

La recogida de datos se realizó de enero a mayo de 2015. Se usaron como estrategia de búsqueda las diferentes combinaciones entre las variables y los operadores Booleanos "AND" y "OR". Las variables de partida utilizadas fueron: Cancer, vascular devices, complications, Port-A-Cath, PICC line, chemotherapy, oncology patient, implantable port, infections, thrombosis, extravasation, CVC management, life quality.

La selección de artículos se realizó mediante la lectura del título y/o el resumen y posteriormente, mediante la aplicación de los criterios de inclusión y exclusión.

Se realizó un análisis descriptivo y temático categorial de los datos obtenidos.

\section{RESULTADOS}

En principio, se seleccionaron un total de 124 artículos utilizando los criterios de búsqueda anteriormente descritos. La Tabla I refleja el proceso de descarte y de selección de algunos de estos artículos en base a los objetivos propuestos en el estudio.

Tabla I: Proceso de selección de artículos de las diferentes bases de datos

\begin{tabular}{lcccc}
\hline BASES DE DATOS & $\begin{array}{c}\text { Art. } \\
\text { seleccionados } \\
\text { por título y } \\
\text { resumen }\end{array}$ & $\begin{array}{c}\text { Art. descartados } \\
\text { por imposibilidad de } \\
\text { adquirir texto } \\
\text { completo }\end{array}$ & $\begin{array}{c}\text { Art. descartados } \\
\text { por inutilidad } \\
\text { sobre la temática }\end{array}$ & Art. útiles \\
$\begin{array}{l}\text { PUBMED } \\
\text { S2 }\end{array}$ & 24 & 16 & 10 \\
GOOGLE ACADÉMICO & 37 & 9 & 19 & 8 \\
$\begin{array}{l}\text { HEALTH \& MEDICAL } \\
\text { COMPLETE }\end{array}$ & 26 & 5 & 12 & 11 \\
SCIELO & 9 & 5 & 1 & 3 \\
OTROS & - & - & 48 & 10 \\
Total: & $\mathbf{1 2 4}$ & $\mathbf{4 3}$ & $\mathbf{4 8}$ \\
\hline
\end{tabular}


Finalmente, se realizó un análisis descriptivo de un total de 42 artículos. La Tabla II recoge los diferentes tipos de estudios y documentos que han resultado de la revisión sistemática.

Tabla II. Tipos de documentos adquiridos El tipo de documento "Otros" abarca la Estudios observacionales información adquirida de Sedes Webs, - Retrospectivos $17 \quad$ mediante el uso del buscador Google, y por

- Prospectivos tanto, no han sido adquiridos en bases de datos.

\begin{tabular}{|c|c|c|c|c|c|}
\hline \multicolumn{2}{|c|}{ Revisiones bibliográficas } & \multicolumn{4}{|c|}{ Los estudios observacionales son los } \\
\hline Guías & \multicolumn{5}{|c|}{ 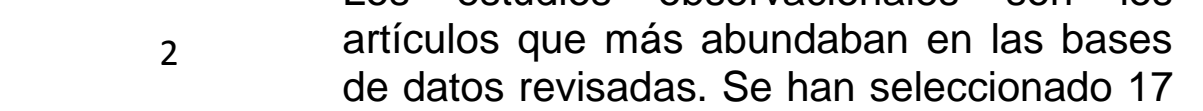 } \\
\hline Otros & 10 & \multicolumn{4}{|c|}{$\begin{array}{l}\text { estudios retrospectivos y } 6 \text { prospectivos, } \\
\text { todos ellos revisados v validados por la }\end{array}$} \\
\hline Total & 42 & \multirow{2}{*}{\multicolumn{4}{|c|}{$\begin{array}{l}\text { declaración STROBE (Anexo I), una } \\
\text { herramienta que tiene el objetivo de } \\
\text { estudios observacionales en epidemiología. Algunos } \\
\text { eflejados en la Tabla III. }\end{array}$}} \\
\hline $\begin{array}{l}\text { rtalecer la prese } \\
\text { atos de estos est }\end{array}$ & $\begin{array}{l}\text { ón de los } \\
\text { quedan re }\end{array}$ & & & & \\
\hline \multicolumn{6}{|c|}{ Tabla III: Datos sobre los estudios observacionales retrospectivos y prospectivos $1 / 2$} \\
\hline Autor/es & $\begin{array}{l}\text { País; } \\
\text { Año de } \\
\text { publicación }\end{array}$ & $\begin{array}{l}\text { Tipo de } \\
\text { estudio }\end{array}$ & $\begin{array}{l}\text { Fechas de } \\
\text { estudio de la } \\
\text { muestra }\end{array}$ & Muestra & $\begin{array}{l}\text { Validado } \\
\text { por } \\
\text { checklist }\end{array}$ \\
\hline Cortés-Flores AO et al. & $\begin{array}{l}\text { México; } \\
2012\end{array}$ & $\begin{array}{l}\text { Revisión } \\
\text { retrospectiva }\end{array}$ & $\begin{array}{l}\text { Mayo 2008-Enero } \\
2012\end{array}$ & 156 & $\begin{array}{l}\text { STROBE } \\
17 / 22\end{array}$ \\
\hline Alvarez JA et al. & $\begin{array}{l}\text { Paraguay; } \\
2012\end{array}$ & $\begin{array}{l}\text { Estudio } \\
\text { retrospectivo no } \\
\text { aleatorio }\end{array}$ & $\begin{array}{l}\text { Marzo } 2011- \\
\text { Junio } 2012\end{array}$ & 60 & $\begin{array}{l}\text { STROBE } \\
18 / 22\end{array}$ \\
\hline Ince $\mathrm{E}$ et al. & $\begin{array}{l}\text { Turquía; } \\
2014\end{array}$ & $\begin{array}{l}\text { Estudio } \\
\text { retrospectivo }\end{array}$ & $\begin{array}{l}\text { Enero 2005- } \\
\text { Diciembre } 2013\end{array}$ & 108 & $\begin{array}{l}\text { STROBE } \\
16 / 22\end{array}$ \\
\hline Kim JT et al. & Corea; 2012 & $\begin{array}{l}\text { Estudio } \\
\text { retrospectivo }\end{array}$ & $\begin{array}{l}\text { Noviembre2004- } \\
\text { Enero } 2008\end{array}$ & 442 & $\begin{array}{l}\text { STROBE } \\
16 / 22\end{array}$ \\
\hline Bassi KK et al. & India; 2012 & $\begin{array}{l}\text { Revisión } \\
\text { retrospectiva }\end{array}$ & $\begin{array}{l}\text { Enero2009- } \\
\text { Marzo-2011 }\end{array}$ & 81 & $\begin{array}{l}\text { STROBE } \\
15 / 22\end{array}$ \\
\hline Granziera E et al. & $\begin{array}{l}\text { Italia; } \\
2014\end{array}$ & $\begin{array}{l}\text { Estudio } \\
\text { retrospectivo }\end{array}$ & $\begin{array}{l}\text { Noviembre2006 } \\
\text {-Noviembr2011 }\end{array}$ & 796 & $\begin{array}{l}\text { STROBE } \\
17 / 22\end{array}$ \\
\hline Shim J et al. & Corea; 2014 & $\begin{array}{l}\text { Estudio } \\
\text { retrospectivo }\end{array}$ & $\begin{array}{l}\text { Agosto } 2003- \\
\text { Noviembre2011 }\end{array}$ & 1747 & $\begin{array}{l}\text { STROBE } \\
19 / 22\end{array}$ \\
\hline Seok JP et al. & Corea; 2014 & $\begin{array}{l}\text { Estudio } \\
\text { retrospectivo }\end{array}$ & $\begin{array}{l}\text { Diciembre 2008- } \\
\text { Marzo } 2013\end{array}$ & 156 & $\begin{array}{l}\text { STROBE } \\
15 / 22\end{array}$ \\
\hline $\begin{array}{l}\text { Barroso de Oliveira E } \\
\text { et al. }\end{array}$ & $\begin{array}{l}\text { Brasil; } \\
2013\end{array}$ & $\begin{array}{l}\text { Estudio } \\
\text { retrospectivo }\end{array}$ & $\begin{array}{l}\text { Marzo 2005- } \\
\text { Septiembr2009 }\end{array}$ & 793 & $\begin{array}{l}\text { STROBE } \\
17 / 22\end{array}$ \\
\hline
\end{tabular}


Tabla III: Datos sobre los estudios observacionales retrospectivos y prospectivos $2 / 2$

\begin{tabular}{|c|c|c|c|c|c|}
\hline Autor/es & $\begin{array}{l}\text { País; } \\
\text { Año de } \\
\text { publicación }\end{array}$ & Tipo de estudio & $\begin{array}{l}\text { Fechas de } \\
\text { estudio de la } \\
\text { muestra }\end{array}$ & Muestra & $\begin{array}{l}\text { Validado } \\
\text { por } \\
\text { checklist }\end{array}$ \\
\hline Paleczny J et al. & Polonia; 2013 & $\begin{array}{l}\text { Estudio } \\
\text { prospectivo }\end{array}$ & $\begin{array}{l}\text { Abril 2008- Mayo } \\
2013\end{array}$ & 220 & $\begin{array}{l}\text { STROBE } \\
19 / 22\end{array}$ \\
\hline D'Souza PC et al. & $\begin{array}{l}\text { Omán; } \\
2014\end{array}$ & $\begin{array}{l}\text { Estudio } \\
\text { retrospectivo }\end{array}$ & $\begin{array}{l}\text { Enero } 2007- \\
\text { Febrero } 2013\end{array}$ & 106 & $\begin{array}{l}\text { STROBE } \\
16 / 22\end{array}$ \\
\hline Baiocco GG et al. & $\begin{array}{l}\text { Brasil; } \\
2010\end{array}$ & $\begin{array}{l}\text { Estudio } \\
\text { retrospectivo }\end{array}$ & $2000-2007$ & 231 & $\begin{array}{l}\text { STROBE } \\
19 / 22\end{array}$ \\
\hline Cotogni P et al. & $\begin{array}{l}\text { Italia; } \\
2015\end{array}$ & $\begin{array}{l}\text { Estudio } \\
\text { prospectivo }\end{array}$ & $\begin{array}{l}\text { Junio 2008- Mayo } \\
2013\end{array}$ & 250 & $\begin{array}{l}\text { STROBE } \\
18 / 22\end{array}$ \\
\hline Ahn DH et al. & $\begin{array}{l}\text { EEUU; } \\
2013\end{array}$ & $\begin{array}{l}\text { Estudio } \\
\text { retrospectivo }\end{array}$ & $\begin{array}{l}\text { Enero 2006- } \\
\text { Diciembre } 2009\end{array}$ & 237 & $\begin{array}{l}\text { STROBE } \\
18 / 22\end{array}$ \\
\hline Hatakeyama $\mathrm{N}$ et al. & $\begin{array}{l}\text { Japón; } \\
2011\end{array}$ & $\begin{array}{l}\text { Revisión } \\
\text { retrospectiva }\end{array}$ & $\begin{array}{l}\text { Octubre 2001- } \\
\text { Abril } 2010\end{array}$ & 78 & $\begin{array}{l}\text { STROBE } \\
16 / 22\end{array}$ \\
\hline Liu Y et al. & $\begin{array}{l}\text { China; } \\
2015\end{array}$ & $\begin{array}{l}\text { Estudio } \\
\text { prospectivo }\end{array}$ & $\begin{array}{l}\text { Mayo } 2010- \\
\text { Febrero } 2013\end{array}$ & 311 & $\begin{array}{l}\text { STROBE } \\
17 / 22\end{array}$ \\
\hline Coady K et al. & $\begin{array}{l}\text { Reino Unido; } \\
2015\end{array}$ & $\begin{array}{l}\text { Estudio } \\
\text { prospectivo }\end{array}$ & $\begin{array}{l}\text { Enero 2011- } \\
\text { Agosto } 2013\end{array}$ & 55 & $\begin{array}{l}\text { STROBE } \\
18 / 22\end{array}$ \\
\hline Patel GS et al. & $\begin{array}{l}\text { Australia; } \\
2014\end{array}$ & $\begin{array}{l}\text { Estudio } \\
\text { prospectivo }\end{array}$ & $\begin{array}{l}\text { Diciembre 2004- } \\
\text { Enero } 2010\end{array}$ & 70 & $\begin{array}{l}\text { STROBE } \\
16 / 22\end{array}$ \\
\hline Matsuzaki M et al. & $\begin{array}{l}\text { Japón; } \\
2006\end{array}$ & $\begin{array}{l}\text { Revisión } \\
\text { retrospectiva }\end{array}$ & $\begin{array}{l}\text { Junio 2001- } \\
\text { Diciembre } 2004\end{array}$ & 53 & $\begin{array}{l}\text { STROBE } \\
16 / 22\end{array}$ \\
\hline Kabalan P et al. & $\begin{array}{l}\text { Chile; } \\
2010\end{array}$ & $\begin{array}{l}\text { Revisión } \\
\text { retrospectiva } \\
\text { descriptiva }\end{array}$ & $\begin{array}{l}\text { Enero 2005- } \\
\text { Diciembre } 2007\end{array}$ & 59 & $\begin{array}{l}\text { STROBE } \\
19 / 22\end{array}$ \\
\hline Alfaro-Rubio A et al. & $\begin{array}{l}\text { España; } \\
2006\end{array}$ & $\begin{array}{l}\text { Estudio } \\
\text { prospectivo }\end{array}$ & $\begin{array}{l}\text { Enero } 2000- \\
\text { Diciembre } 2003\end{array}$ & 2186 & $\begin{array}{l}\text { STROBE } \\
16 / 22\end{array}$ \\
\hline Romaniello HO et al. & $\begin{array}{l}\text { Argentina; } \\
2005\end{array}$ & $\begin{array}{l}\text { Estudio } \\
\text { retrospectivo }\end{array}$ & $\begin{array}{l}\text { Mayo 1998- } \\
\text { Junio } 2000\end{array}$ & 492 & $\begin{array}{l}\text { STROBE } \\
18 / 22\end{array}$ \\
\hline Nagel SN et al. & $\begin{array}{l}\text { Alemania; } \\
2011\end{array}$ & $\begin{array}{l}\text { Estudio } \\
\text { retrospectivo }\end{array}$ & $\begin{array}{l}\text { Enero 2008- } \\
\text { Enero } 2010\end{array}$ & 98 & $\begin{array}{l}\text { STROBE } \\
15 / 22\end{array}$ \\
\hline
\end{tabular}

Por otro lado, las revisiones bibliográficas obtenidas han sido valoradas por la declaración PRISMA (Anexo II), una propuesta para mejorar la publicación de revisiones sistemáticas y metaanálisis. La Tabla IV refleja algunos datos de estas revisiones. 
Tabla IV: Datos sobre las revisiones bibliográficas

\begin{tabular}{|c|c|c|c|}
\hline Autor/es & $\begin{array}{c}\text { País; } \\
\text { Año de publicación }\end{array}$ & Temática & $\begin{array}{l}\text { Validado por } \\
\text { checklist }\end{array}$ \\
\hline Freire E et al. & $\begin{array}{l}\text { España; } 2008 \\
\text { mantenimiento }\end{array}$ & $\begin{array}{l}\text { Descripción de la técnica de } \\
\text { implante del Port-A-Cath, el } \\
\text { del dispositivo } \\
\text { y sus complicaciones. }\end{array}$ & $\begin{array}{c}\text { PRISMA } \\
10 / 27\end{array}$ \\
\hline Gallieni M et al. & Italia; 2008 & $\begin{array}{l}\text { Dispositivos de acceso venoso } \\
\text { disponibles, problemas más } \\
\text { relevantes en relación con su } \\
\text { inserción y su manejo }\end{array}$ & $\begin{array}{c}\text { PRISMA } \\
10 / 27\end{array}$ \\
\hline Kurul S et al. & Turquía; 2002 & $\begin{array}{l}\text { Procedimientos de inserción y } \\
\text { complicaciones del Port-A-Cath, } \\
\text { con énfasis en los problemas } \\
\text { locales y herida de extravasación }\end{array}$ & $\begin{array}{c}\text { PRISMA } \\
12 / 27\end{array}$ \\
\hline Baskin JL et al. & EEUU; 2009 & $\begin{array}{l}\text { Diagnóstico, manejo y prevención } \\
\text { de oclusiones y trombosis de los } \\
\text { catéteres venosos centrales }\end{array}$ & $\begin{array}{c}\text { PRISMA } \\
13 / 27\end{array}$ \\
\hline Leonidou L et al. & Grecia; 2010 & $\begin{array}{l}\text { Epidemiología, patogénesis, } \\
\text { diagnóstico, microbiología y } \\
\text { manejo de las infecciones } \\
\text { relacionadas con el catéter }\end{array}$ & $\begin{array}{c}\text { PRISMA } \\
10 / 27\end{array}$ \\
\hline Warren P et al. & EEUU; 2011 & $\begin{array}{l}\text { Indicaciones para el tratamiento } \\
\text { complicaciones y éxitos del } \\
\text { tratamiento de la trombosis y } \\
\text { el síndrome de la vena cava } \\
\text { superior }\end{array}$ & $\begin{array}{l}\text { PRISMA } \\
10 / 27\end{array}$ \\
\hline Sauerland C et al. & EEUU; 2006 & $\begin{array}{l}\text { Incidencia, recomendaciones y } \\
\text { manejo de Enfermería relevantes } \\
\text { a la extravasación vesicante }\end{array}$ & $\begin{array}{l}\text { PRISMA } \\
10 / 27\end{array}$ \\
\hline
\end{tabular}

Como se indicó previamente, la realización de este trabajo está orientada a conocer las complicaciones de la administración de quimioterapia con el uso de diferentes dispositivos de acceso venoso central. Las principales temáticas de los 42 documentos están relacionadas con los "Port- $A$-Cath" (15 artículos), "CCIP" (10 artículos), "Port-A-Cath + CCIP" (6 artículos) y "Otros" (11 artículos).

De esta manera, se hace evidente que la mayoría de los documentos que se han seleccionado abarcan el Port-A-Cath en su temática principal, por lo que se demuestra que la producción científica de dicho dispositivo es mayor que la del CCIP.

Son pocos los estudios comparativos que abarcan ambos dispositivos; la mayoría de documentos que proporcionan información de ambos CVC son revisiones y guías. 
Cabe comentar que la temática "Otros" recoge aquellos artículos que no tratan de los dispositivos Port-A-Cath o los CCIP como tal, sino que estudian los CVC en general o alguna de sus complicaciones sin relacionarlas con un tipo de catéter en concreto.

El análisis temático categorial produjo la siguiente información:

1- Complicaciones más frecuentes: Infecciones; Trombosis y oclusión del catéter.

2- Otras complicaciones: Fractura del catéter y embolismo; Migración o dislocación del catéter; Extravasación; Flebitis; Necrosis de la piel.

3- Calidad de vida.

\section{DISCUSIÓN}

\section{Complicaciones más frecuentes}

\section{Infecciones}

Las infecciones son las complicaciones más frecuentes de los Port-A-Cath, como han demostrado algunos estudios observacionales de pacientes con este tipo de dispositivo implantado ${ }^{(7,9,10,11,12,13,14)}$. Los pacientes oncológicos son más propensos a ellas que otro tipo de paciente debido a su inmunosupresión ${ }^{(15)}$.

En un estudio, el 6,17\% de los pacientes con Port-A-Cath padeció una infección sistémica, mientras que el $2,4 \%$, una infección del bolsillo subcutáneo ("pocket infection" en inglés), es decir, una acumulación de líquido infectado en el tejido subcutáneo que rodea al dispositivo ${ }^{(16)}$. Esto es, son frecuentes tanto las infecciones sistémicas como locales.

Por otro lado, las infecciones relacionadas con los CCIP también son las más frecuentes junto con la trombosis. Un 13,9\% de los 237 CCIP estudiados por Ahn DH et al. ${ }^{(17)}$ padecieron una infección relacionada con el catéter, así como en otro estudio, donde hubo 28 casos de infecciones sistémicas y 6 de infección del sitio de salida del catéter de los 93 CCIP estudiados ${ }^{(18)}$, siendo éstas unas cifras elevadas.

La retirada del catéter, la administración de una solución antimicrobiana de sellado del catéter ("lock" terapia) y el comienzo del tratamiento antibiótico sistémico son algunos procedimientos para el manejo de estas infecciones ${ }^{(15,19)}$.

La retirada del dispositivo es una alternativa que se ha de considerar. En el caso de los CCIP, el catéter debe ser retirado cuando hay una complicación infecciosa o sospecha de ella. Al contrario ocurre con los Port-A-Cath, ya que se deben evaluar algunos factores para su retirada (evaluación de la gravedad del estado de salud del paciente, una prueba de que el catéter está infectado, disponibilidad de canalizar otro acceso venoso, evaluación del patógeno y presencia de otras complicaciones

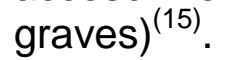

El tratamiento antibiótico sistémico debe comenzarse en todos los casos y, en ocasiones, podría asociarse con la "lock" terapia, en la cual los antibióticos usados deben ser compatibles con el catéter usado y con otros componentes de la terapia, como son la heparina, el suero fisiológico y otros antibióticos ${ }^{(19,20)}$. 
Por otro lado, las infecciones locales del reservorio (infección del bolsillo subcutáneo) pueden tratarse con antibióticos de carácter local; los 3 casos de infección del bolsillo subcutáneo que se registraron en el estudio de Kim JT et al. ${ }^{(21)}$ se curaron con éxito tras la administración de tratamiento local.

Los CVC implantados quirúrgicamente parecen tener una cuarta parte del riesgo de infección que se asocia a los CVC insertados percutáneamente, es decir, los CCIP tienen un mayor riesgo ${ }^{(19)}$.

A pesar de esto, un estudio comparativo de ambos dispositivos concluye lo contrario. Aunque el tamaño de la muestra de este estudio no es muy grande y haya ocurrido un solo caso de infección, este caso es de un paciente con Port-A-Cath contra ninguno de infección del $\operatorname{CCIP}^{(5)}$.

\section{Trombosis y oclusión del catéter}

Se afirma que hay una obstrucción del catéter cuando es difícil o imposible la extracción de sangre o la infusión de medicamentos ${ }^{(13)}$. Sin embargo, la obstrucción de estos catéteres no solo es consecuencia de una trombosis, sino también puede ser una oclusión por fallos mecánicos (acodamiento del catéter, suturas demasiado apretadas, bloqueo de la punta del catéter por la pared del vaso sanguíneo, malposición de la aguja en la membrana del reservorio del Port-A-Cath o el síndrome "pinch-off", explicado más adelante) $)^{(22)}$.

Un estudio acerca de las complicaciones de los Port-A-Cath registró un $6,17 \%$ de obstrucciones trombóticas del catéter frente a un 3,6\% de obstrucciones no trombóticas ${ }^{(16)}$, lo que demuestra que son más frecuentes las oclusiones relacionadas con trombosis.

También es frecuente que este tipo de pacientes desarrollen trombosis venosa, es decir, la formación de un trombo cerca de un $\mathrm{CVC}^{(22)}$. Además, este tipo de trombosis puede desarrollarse en grandes vasos. En un estudio se registraron 20 casos de trombosis venosa relacionados con Port-a-Cath en la vena subclavia, yugular interna y un caso de trombosis de la Vena Cava Superior (VCS), siendo éste motivo de retirada de 10 de los dispositivos ${ }^{(13)}$.

Cabe decir que la oclusión de la VCS se conoce como "Síndrome de la Vena Cava Superior", mediante el cual, se obstruye el flujo sanguíneo de salida de la cabeza y las extremidades superiores. Esta oclusión puede ser causa de la expansión del tumor, que invade u oprime la vena, pero también podría ser debido a la formación de un trombo relacionado con un dispositivo endovascular ${ }^{(23)}$.

Los CCIP resultan más propensos al desarrollo de complicaciones trombóticas $u$ oclusivas que los Port-A-Cath. Un estudio prospectivo registró un $51,4 \%$ de casos de trombosis de su muestra (311 pacientes con un CCIP in situ) ${ }^{(24)}$, una cifra muy elevada.

Otro estudio que compara ambos dispositivos, 4 casos de trombosis de las venas profundas en pacientes con un CCIP implantado, junto con 3 casos de obstrucción del catéter, afirman que este dispositivo se predispone en mayor medida al desarrollo de estas complicaciones que los Port-A-Cath que, por el contrario, no registraron ningún $\operatorname{caso}^{(5)}$. 
Para la desobstrucción de la luz del catéter, hay autores que, tras la realización de una revisión, declaran que se debe usar una jeringa de $10 \mathrm{~mL}$ con la solución más apropiada según el tipo de obstrucción ${ }^{(15)}$. En caso de obstrucción trombótica, éstos también afirman que debe usarse medicación trombolítica en casos agudos, mientras que en los casos con sintomatología crónica, se debe usar heparina de bajo peso molecular combinada con anticoagulantes orales o únicamente la heparina a largo plazo $^{(15)}$.

Con la documentación analizada se observa que hay discrepancias sobre qué solución y qué diluciones de heparina son las más adecuadas para el sellado de los CVC. Una revisión y una guía de práctica clínica coinciden en que para ambos dispositivos el sellado se lleva a cabo con 10 o $20 \mathrm{~mL}$ de suero fisiológico seguido de $5 \mathrm{~mL}$ de heparina $(10 \mathrm{U} / \mathrm{mL}$ para el lavado diario o $100 \mathrm{U} / \mathrm{ml}$ en el caso de intervalos más largos) ${ }^{(15,25)}$.

Sin embargo, otras guías afirman que el sellado de un CCIP debe realizarse únicamente con suero fisiológico hasta que no haya más evidencia o se indique lo contrario $^{(26)}$, y el sellado del Por-A-Cath, con $5 \mathrm{~mL}$ de suero heparinizado ${ }^{(27)}$.

Por otra parte, estas complicaciones pueden ser prevenidas con apropiados cuidados de enfermería: el sellado, tanto del Port-A-Cath como del CCIP, tras la administración de medicamentos, trasfusiones o extracciones de sangre y cuando no va a ser usado es crucial para el mantenimiento de su permeabilidad y eficacia.

Las indicaciones principales de retirada de estos dispositivos en caso de trombosis son: la obstrucción irreversible de la luz del catéter, la infección del trombo y la malposición de la punta del catéter ${ }^{(15)}$.

\section{Otras complicaciones}

\section{Fractura del catéter y embolismo (Síndrome “pinch-off”)}

La rotura y el embolismo del catéter del Port-A-Cath pueden causar serias complicaciones, a pesar de que sea una complicación infrecuente $(0,1 \%)^{(28)}$. En cuatro estudios sobre este dispositivo, solo había un caso de esta complicación en cada uno ${ }^{(6,12,14,21)}$.

La ruptura del reservorio es dolorosa y crea un hematoma ${ }^{(29)}$; en cuanto a la membrana, su rotura es muy poco probable, ya que esta puede ser puncionada alrededor de mil veces sin ser dañada ${ }^{(28)}$.

Por el contrario, los CCIP parecen fracturarse con mayor asiduidad. En un estudio de 109 de estos catéteres, se dieron 7 casos de rotura ${ }^{(30)}$, además de otro estudio que compara los CCIP con los Por-A-Cath, registrando 2 casos de ruptura del catéter contra un caso respectivamente ${ }^{(5)}$. Una medida de prevención de ruptura del catéter de estos dispositivos podría ser la identificación de la posibilidad del síndrome "pinchoff', es decir, la posibilidad de que el catéter pudiera comprimirse entre la clavícula y la primera costilla ${ }^{(15,22,28)}$. Este síndrome podría traer consigo malfuncionamiento, obstrucción, daño y, como ya se ha menconado, fractura del catéter con embolismo $^{(15)}$.

La retirada del dispositivo está indicada en esta complicación. Algunos estudios con pacientes que sufrieron fractura y embolismo de la punta del catéter, indican que el 
dispositivo tuvo que ser retirado y la extracción del fragmento se realizó mediante cateterismo cardíaco ${ }^{(6,21)}$.

\section{Migración o dislocación del catéter}

Normalmente, la dislocación de los CCIP es secundaria a la inapropiada fijación del catéter (mediante suturas o apósitos) o por unos cuidados enfermeros inadecuados de la zona de salida del catéter ${ }^{(15)}$.

La dislocación de los Port-A-Cath puede suceder si el bolsillo subcutáneo es muy grande y el reservorio no se une al tejido subyacente ${ }^{(29)}$, pero la migración del reservorio no suele ocurrir con demasiada frecuencia: de todos los estudios retrospectivos revisados de pacientes con CVC totalmente implantado, solo uno de ellos ha registrado 4 casos de migración en su serie ${ }^{(11)}$. Sin embargo, en otro estudio un $7,1 \%$ de casos (19 pacientes) sufrieron un desplazamiento de sus $\operatorname{CCIP}^{(31)}$.

Además, 2 casos de desplazamiento parcial del catéter del CCIP frente a ninguno del Port-A-Cath en un estudio comparativo demuestran una mayor incidencia de esta complicación en los $\mathrm{CCIP}^{(5)}$.

\section{Extravasación}

La extravasación es la fuga o el escape de algún medicamento de la vena al tejido perivascular ${ }^{(32,33)}$. Las consecuencias de la extravasación oscilan desde molestias hasta destrucción severa del tejido. La agresividad depende del tipo de droga que se haya fugado: no vesicante, irritante o vesicante ${ }^{(32,33)}$.

Pueden usarse medidas no farmacológicas para la reducción de las molestias y la inflamación producida por la extravasación de las drogas no vesicantes e irritantes. Sin embargo, la fuga de una droga vesicante puede causar un serio daño tisular ${ }^{(29,32,33)}$.

La necrosis tisular de la zona es una de estas posibles consecuencias ${ }^{(15,32,33)}$. La herida de extravasación suele ocurrir entre el $0,1 \%$ y $6,5 \%$ de los $\operatorname{casos}^{(15)}$.

Siempre que sea posible, la medicación vesicante debe administrarse mediante un CVC, ya que la vía periférica está asociada a un alto riesgo de extravasación, infiltración y flebitis ${ }^{(15,33,34)}$.

La incidencia de extravasación en Port-A-Cath se encuentra entre $0,3 \%-4,7 \%{ }^{(32)}$. La incorrecta punción de la aguja suele ser la causa más frecuente de extravasación en los Port-A-Cath, como los 3 casos ocurridos en el estudio retrospectivo de Seok JP et al. ${ }^{(12)}$. Por otro lado, un estudio de 607 CCIP registra una incidencia de extravasación del $1,3 \%{ }^{(35)}$. Por tanto, esta complicación es inusual en ambos dispositivos.

En la prevención de estas complicaciones, diferentes autores afirman que es crucial la fijación de la aguja del Port-A-Cath para prevenir su desplazamiento, que debe comprobarse si ha ocurrido una migración del CCIP midiendo la longitud del catéter externo y comparándola con la original y que, además, se debe realizar aspiración de sangre que afirme que el catéter se encuentra en vena y no comenzar el tratamiento si su posición está en duda ${ }^{(32,34)}$. 
En caso de que haya ocurrido extravasación, los autores indican cesar la infusión de drogas inmediatamente y realizar una aspiración del catéter para la posible eliminación de agente vesicante ${ }^{(15,34)}$. En el caso del Port a Cath, aplicar la droga residual desde la aguja e inyectar el antídoto, si está indicado, mientras que, en el caso de los CCIP, usar una aguja para aspirar la droga residual del tejido subcutáneo alrededor de la zona de extravasación e inyectar el antídoto, si está indicado(34). Aplicar frío o calor según el tipo de agente vesicante extravasado y, en caso de necrosis de la piel, intervenir al paciente quirúrgicamente inmediatamente para el desbridamiento y reconstrucción ${ }^{(15,34)}$.

\section{Flebitis}

La flebitis es una inflamación de la vena, cuyos principales síntomas son rojez, calor y sensibilidad a lo largo de la vena por encima de la zona de inserción ${ }^{(36)}$.

Es una complicación más frecuente en los CCIP, debido a que el catéter recorre una de las principales venas del brazo. Dos de los estudios revisados, presentaron algún paciente con esta complicación: un solo caso, en uno de los estudios ${ }^{(18)}$ y 3 , en otro $^{(30)}$.

El protocolo enfermero del Hospital Universitario Ramón y Cajal sobre los CCIP afirma que entre los cuidados de dichos catéteres, se encuentra la vigilancia de aparición de flebitis, la cual deberá realizarse valorando su grado según la escala visual de valoración de flebitis o escala de Maddox ${ }^{(37,38)}$.

Algunas medidas para el manejo de esta complicación podrían ser la elevación de la extremidad, el fomento de ejercicios moderados y la colocación de compresas frías ${ }^{(37)}$. Sin embargo, si el paciente presenta un grado alto de flebitis, se debe considerar la posibilidad de retirada del CCIP $^{(37)}$, solución dada a esta complicación en los estudios mencionados $^{(18,30)}$.

\section{Necrosis de la piel circundante al dispositivo (Port-A-Cath)}

La necrosis de la piel que rodea el reservorio del Port-A-Cath puede ocurrir por diversos motivos. La implantación superficial del dispositivo en quirófano o la delgadez del tejido subcutáneo del paciente pueden ser razones para su desarrollo ${ }^{(15,29)}$.

En un estudio, fue esta última la razón de la aparición de necrosis: el reservorio erosionó la piel del paciente debido a su delgadez ${ }^{(39)}$. En estos pacientes, el reservorio debe ser colocado bajo el músculo para prevenir la necrosis ${ }^{(29)}$.

Otros estudios registraron algún caso de esta complicación debido a la presión ejercida por el reservorio en la piel suprayacente durante el período de tiempo implantado ${ }^{(9,16)}$. En uno de ellos se eliminó en los siguientes años la aparición de esta complicación en sus pacientes, evitando la extrema disección subcutánea y usando un reservorio más pequeño a la hora de su implantación ${ }^{(9)}$.

A pesar de que esta no es una complicación muy usual (muy pocos casos en los estudios revisados ${ }^{(9,16,39)}$, puede ocurrir en cualquier momento del seguimiento, aunque puede ser más frecuente cuando el paciente ha perdido peso en la última fase de su enfermedad ${ }^{(29)}$, por lo que indican que hay que proceder a la retirada del dispositivo para comenzar la cura de la piel ${ }^{(9,16,39)}$. 


\section{Calidad de vida}

El diagnóstico de un cáncer puede ser difícil de afrontar para el paciente y éste, además, debe someterse a un gran número de terapias agresivas ${ }^{(40)}$ que pueden ser, en ocasiones, de larga duración. Es por esto que es recomendable el uso de CVC para mejorar su calidad de vida.

Los dispositivos de larga duración, como son los CCIP y los Port-A-Cath, podrían reducir o evitar tanto las canalizaciones periféricas continuas, que suponen un aumento del sufrimiento para el paciente ${ }^{(39)}$, como las complicaciones asociadas a la quimioterapia.

La facilidad de implantación y retirada de un CCIP sin necesidad de cirugía y dolor mínimo serían algunas de las ventajas de este catéter ${ }^{(8,25)}$. Sin embargo, a pesar de que su frecuencia de complicaciones es baja, tiene una mayor tasa de trombosis que otros CVC, menor duración del catéter (entre 3-12 meses ${ }^{(15)}$ ) que otros dispositivos, velocidad del flujo más lenta y mayor incidencia de malposición ${ }^{(25)}$.

Por otro lado, las ventajas de la implantación del Port-A-Cath son la duración del dispositivo a largo plazo, la inexistencia de catéter externo, baja incidencia de infección y permite el baño con normalidad, así como su mantenimiento, fácil y poco costoso, que solo requiere un sellado con suero heparinizado al mes ${ }^{(15,25)}$. Además, los pacientes pueden preferir este dispositivo por su atracción estética, ya que, al ser totalmente implantado, no queda ningún catéter a la vista, pudiendo preservar la autoestima del paciente, sin que éste pudiera interferir en las actividades de su vida diaria $^{(25)}$.

Su principal desventaja es la necesidad de implantación y retirada mediante una intervención quirúrgica, lo que puede suponer un aumento de ansiedad y temor al paciente, y su coste económico más elevado ${ }^{(15,25)}$.

Se realizaron dos estudios que evaluaban la satisfacción y la calidad de vida de pacientes con un Port-A-Cath implantado mediante un cuestionario, que fue contestado por 42 pacientes $^{(41)}$, en uno de los estudios, y 65 pacientes ${ }^{(42)}$, en otro. Ambos cuestionarios coinciden en que el Port-A-Cath es uno de los CVC que proporciona mayor calidad de vida a los pacientes, ya que la gran mayoría de los encuestados contestó que se encontraban satisfechos con el dispositivo. Aunque en uno de los cuestionarios, el $6,1 \%$ de los pacientes no quedaron muy satisfechos con el efecto estético ${ }^{(42)}$.

Los resultados estéticos no se deben pasar por alto; la imagen es muy importante para la autoestima de los pacientes. Se encuentra estadísticamente probado que el resultado estético de la implantación del dispositivo es un predictor de la satisfacción y la calidad de vida ${ }^{(41)}$.

Sin embargo, los CCIP no tienen estos beneficios para la imagen del paciente ya que parte del catéter se encuentra externo. Además, aunque ambos dispositivos tengan una baja tasa de complicaciones, los CCIP parecen ser más propensos que los PortA-Cath tanto a trombosis como a complicaciones mecánicas, oclusiones, migraciones o fracturas del catéter.

A pesar de esto, la elección de un tipo de catéter u otro depende de varios factores, por lo que no se puede afirmar que uno de ellos proporcione más calidad de vida que 
otro, esto dependerá del tipo de tratamiento a administrar, su duración y el tipo de paciente:

- Los Port-A-Cath son más recomendables en niños y en pacientes que requieran un largo uso de un acceso venoso (más de 2 ó 3 meses) ${ }^{(15)}$.

- Los CCIP se recomiendan para terapias ambulatorias cuando se prevé un uso a medio plazo (3 meses) ${ }^{(15)}$.

\section{CONCLUSIONES}

Los resultados de la revisión sistemática han hecho evidente que la producción científica de los Port-A-Cath es mayor que de los CCIP. Además, los estudios observacionales retrospectivos de pacientes con estos dispositivos implantados son los más abundantes en las bases de datos, por lo que la escasez de ensayos clínicos útiles puede ser una limitación de esta revisión.

Tras el estudio de la literatura existente, se llegó a la conclusión de que las complicaciones más frecuentes de estos dispositivos son las infecciones y las trombosis, aunque los CCIP parecen ser más propensos que los Port-A-Cath tanto a complicaciones trombóticas, como a complicaciones mecánicas tales como oclusiones, migraciones o fracturas del catéter. Una de las limitaciones encontradas durante la realización de la revisión han sido las abundantes publicaciones sobre el tema asociadas a una patología o a un tipo de paciente en concreto, de manera que algunos resultados puedan estar relacionados con el tipo de enfermedad, la edad del paciente, etc.

La mayoría de estas complicaciones pueden ser prevenidas ya que se desarrollan debido a errores en la implantación del dispositivo o a unos cuidados inapropiados. Aun así, es difícil determinar qué dispositivo proporciona mayor calidad de vida al paciente dado que ambos son recomendables dependiendo de cada caso. A pesar de esto, los pacientes suelen preferir los Port-A-Cath por su resultado estético.

\section{REFERENCIAS}

1. Cancer.org [Internet]. American Cancer Society; c2015 [citado 4 abr 2015]. Disponible en: http://www.cancer.org/index

2. Cancer.gov [Internet]. National Cancer Institute; c2015 [citado 4 abr 2015]. Disponible en: http://www.cancer.gov/

3. Seom.org [Internet]. Madrid: Sociedad Española de Oncología Médica; [citado 4 abr 2015]. Las Cifras del Cáncer en España 2014; 20. Disponible en: http://www.seom.org/seomcms/images/stories/recursos/Las cifras del cancer 2014. pdf

4. Aecc.es [Internet]. Madrid: Asociación Española Contra el Cáncer; [actualizado 4 abr 2015; citado 5 abr 2015]. Disponible en: https://www.aecc.es/Paginas/PaginaPrincipal.aspx

5. Patel GS, Jain K, Kumar R, Strickland AH, Pellegrini L, Slavotinek J, et al. Comparison of peripherally inserted central venous catheters (PICC) versus subcutaneously implanted port-chamber catheters by complication and cost for patients receiving chemotherapy for non-haematological malignancies. Support Care Cancer. 2014;22(1):121-8. 
6. Cortés-Flores AO, Morgan-Villela G, Juárez-Uzeta EA, Fuentes-Orozco C, JiménezTornero J, González-Ojeda A. Dispositivos de acceso venoso central totalmente implantables en pacientes con cáncer. Experiencia en un Centro Oncológico Privado. Cir Cir 2012;80:429-434.

7. Alvarez JA, Villalba WO, Encina WP, Vega RB. Reservorio venoso subcutáneo. Cir.parag 2012;36(2):14-16.

8. Baiocco GG, da Silva, Jefferson Luis Braga. La utilización del catéter central de inserción periférica (CCIP) en el ambiente hospitalario. Rev. Latino-Am. Enfermagem 2010; 18(6):[07 pantallas].

9. Ince E, Oguzkurt P, Temiz A, Ezer S, Gezer H, Yazici N, et al. Complications of total implantable access ports and efficacy of Taurolidine-citrate lock solution against catheter-related infections. Afr J Paediatr Surg. Apr-Jun 2014;11(2):138-42.

10. Granziera E, Scarpa M, Ciccarese A, Filip B, Cagol M, Manfredi V, et al. Totally implantable venous access devices: retrospective analysis of different insertion techniques and predictors of complications in 796 devices implanted in a single institution. BMC Surgery 2014;14:n/a-27.

11. Shim J, Seo T, Song MG, Cha I, Kim JS, Choi CW, et al. Incidence and risk factors of infectious complications related to implantable venous-access ports. Korean $\mathrm{J}$ Radiol. 2014;15(4):494-500.

12. Seok JP, Kim YJ, Cho HM, Ryu HY, Hwang WJ, Sung TY. A retrospective clinical study: complications of totally implanted central venous access ports. Korean $\mathrm{J}$ Thorac Cardiovasc Surg. 2014;47(1):26-31.

13. de Oliveira EB, Reis MA, Avelar TM, Vieira SC. Totally implantable central venous catheters for chemotherapy: experience with 793 patients. Rev. Col. Bras. Cir. 2013;40(3):186-190

14. D'Souza PC, Kumar S, Kakaria A, Al-Sukaiti R, Zahid KF, Furrukh M, et al. Use of port-a-cath in cancer patients: a single-center experience. J Infect Dev Ctries 2014 2014;8(11):1476-1482

15. Gallieni M, Pittiruti M, Biffi R. Vascular access in oncology patients. CA: CA Cancer J Clin. 2008;58(6):323-346.

16. Bassi KK, Giri AK, Pattanayak M, Abraham SW, Pandey KK. Totally implantable venous access ports: retrospective review of long-term complications in 81 patients. Indian J Cancer. 2012;49(1):114-118.

17. Ahn DH, Illum HB, Wang DH, Sharma A, Dowell JE. Upper extremity venous thrombosis in patients with cancer with peripherally inserted central venous catheters: a retrospective analysis of risk factors. J Oncol Pract. 2013;9(1):e8-12.

18. Hatakeyama N, Hori T, Yamamoto $M$, Mizue $N$, Inazawa N, Igarashi $K$, et al. An evaluation of peripherally inserted central venous catheters for children with cancer requiring long-term venous access. Int J Hematol 2011;94(4):372-7.

19. Leonidou L, Gogos CA. Catheter-related bloodstream infections: catheter management according to pathogen. Int J Antimicrob Agents. 2010;36:S26-S32.

20. Kabalan P, Rodríguez N, Tordecilla J, Sepúlveda F. Infecciones de Catéter Venoso Central y Lock Terapia en Pacientes Oncológicos. Rev Chil Pediatr. 2010;81(5):425-431

21. Kim JT, Oh TY, Chang WH, Jeong YK. Clinical review and analysis of complications of totally implantable venous access devices for chemotherapy. Med Oncol. 2012;29(2):1361-1364.

22. Baskin JL, Pui C, Reiss U, Wilimas JA, Metzger ML, Ribeiro RC, et al. Management of occlusion and thrombosis associated with long-term indwelling central venous catheters. Lancet. 2009;374(9684):159-169. 
23. Warren $P$, Burke $C$. Endovascular management of chronic upper extremity deep vein thrombosis and superior vena cava syndrome. Semin Intervent Radiol. 2011;28(1):32-38.

24. Liu Y, Gao Y, Wei L, Chen W, Ma X, Song L. Peripherally inserted central catheter thrombosis incidence and risk factors in cancer patients: a double-center prospective investigation. Ther Clin Risk Manag. 2015; 11:153-160.

25. Bishop L, Dougherty L, Bodenham A, Mansi J, Crowe P, Kibbler C, et al. Guidelines on the insertion and management of central venous access devices in adults. Int J Lab Hematol. 2007;29(4):261-278.

26. Health.qld.gov.au [Internet].The State of Queensland; c1996-2015 [citado 12 abr 2015]. Guideline Peripherally Inserted Central Venous Catheter (PICC); 18. Disponible en: http://www.health.qld.gov.au/qhpolicy/docs/gdl/qh-gdl-321-6-1.pdf

27. Health.qld.gov.au [Internet].The State of Queensland; c1996-2015 [citado $10 \mathrm{abr}$ 2015]. Guideline Totally Implantable Central Venous Access Ports; 18. Disponible: http://www.health.qld.gov.au/qhpolicy/docs/gdl/qh-gdl-321-6-6.pdf

28. Freire E, De la Iglesia A, Rodríguez C, López M, González M, Peleteiro R, et al. Reservorios venosos centrales totalmente implantables, tipo Port-A-Cath, en pacientes oncológicos: Revisión de Complicaciones. Rev Soc Esp Dolor 2008;7:451462.

29. Kurul S, Saip P, Aydin T. Totally implantable venous-access ports: local problems and extravasation injury. Lancet Oncol 2002 Nov 2002;3(11):684-92.

30. Matsuzaki A, Suminoe A, Koga Y, Hatano M, Hattori S, Hara T. Long-term use of peripherally inserted central venous catheters for cancer chemotherapy in children. Support Care Cancer. 2006;14(2):153-160.

31. Cotogni P, Barbero C, Garrino C, Degiorgis C, Mussa B, De Francesco A, et al. Peripherally inserted central catheters in non-hospitalized cancer patients: 5-year results of a prospective study. Support Care Cancer. 2015;23(2):403-409.

32. Sauerland C, Engelking C, Wickham R, Corbi D. Vesicant extravasation part I: Mechanisms, pathogenesis, and nursing care to reduce risk. Onc Nurs Society. 2006; 33(6):1134-1141.

33. Alfaro-Rubio A, Sanmartín O, Requena C, Llombart B, Botella-Estrada R, Nagore $\mathrm{E}$, et al. Extravasación de agentes citostáticos: una complicación grave del tratamiento oncológico. Actas Dermosifiliogr. 2006;97(3):169-176.

34. Hadaway LC. Preventing extravasation from a central line. Nursing. 2004;34(6):223.

35. Romaniello HO. Incidencia de extravasación del líquido de perfusión por el uso de catéteres percutáneos. Arch.argent.pediatr. 2005;103(1):31-35.

36. Coramhc.com [Internet]. Coram LLC; c2014 [citado 10 abr 2015]. Your Peripherally Inserted Central Catheter (PICC); 6. Disponible en:

http://www.coramhc.com/patientresources/pdf/Your PICC.pdf

37. Madrid.org: Hospital Universitario Ramón y Cajal [Internet]. Comunidad de Madrid; [citado 15 mayo]. Protocolo general Catéteres Venosos Centrales de Inserción

Periférica; 7. Disponible en:

http://www.madrid.org/cs/Satellite?blobcol=urldata\&blobheader=application\%2Fpdf\&bl obkey=id\&blobtable $=$ MungoBlobs\&blobwhere $=1202756185597$ \&ssbinary=true

38. Suárez EG, Martínez ARA, Bueno BM. Protocolo para la inserción, mantenimiento y retirada del catéter periférico. 2013

39. Coady K, Ali M, Sidloff D, Kenningham RR, Ahmed S. A comparison of infections and complications in central venous catheters in adults with solid tumours. J Vasc Access 2015;16(1):38-41. 
40. Seom.org [Internet]. Madrid: Sociedad Española de Oncología Médica; [citado 16 mayo 2015]. Impacto Psicosocial del Cáncer; 3. Disponible en: http://www.seom.org/seomcms/images/stories/recursos/infopublico/publicaciones/revis ta contigo/n 6/psicooncologia.pdf

41. Nagel S, Teichgräber U, Kausche S, Lehmann A. Satisfaction and quality of life: a survey-based assessment in patients with a totally implantable venous port system. Eur J Cancer Care. 2012;21(2):197-204.

42. Paleczny J, Banyś-Jafernik B, Gazurek K, Kierpieć K, Szczerba H, Zipser P. Longterm totally implantable venous access port systems - one center experience. Anaesthesiol Intensive Ther. 2013;45(4):215-222. 


\section{ANEXOS}

\section{Anexo I: Declaración STROBE}

Tabla 1

Declaración STROBE: lista de puntos esenciales que deben describirse en la publicación de estudios observacionales

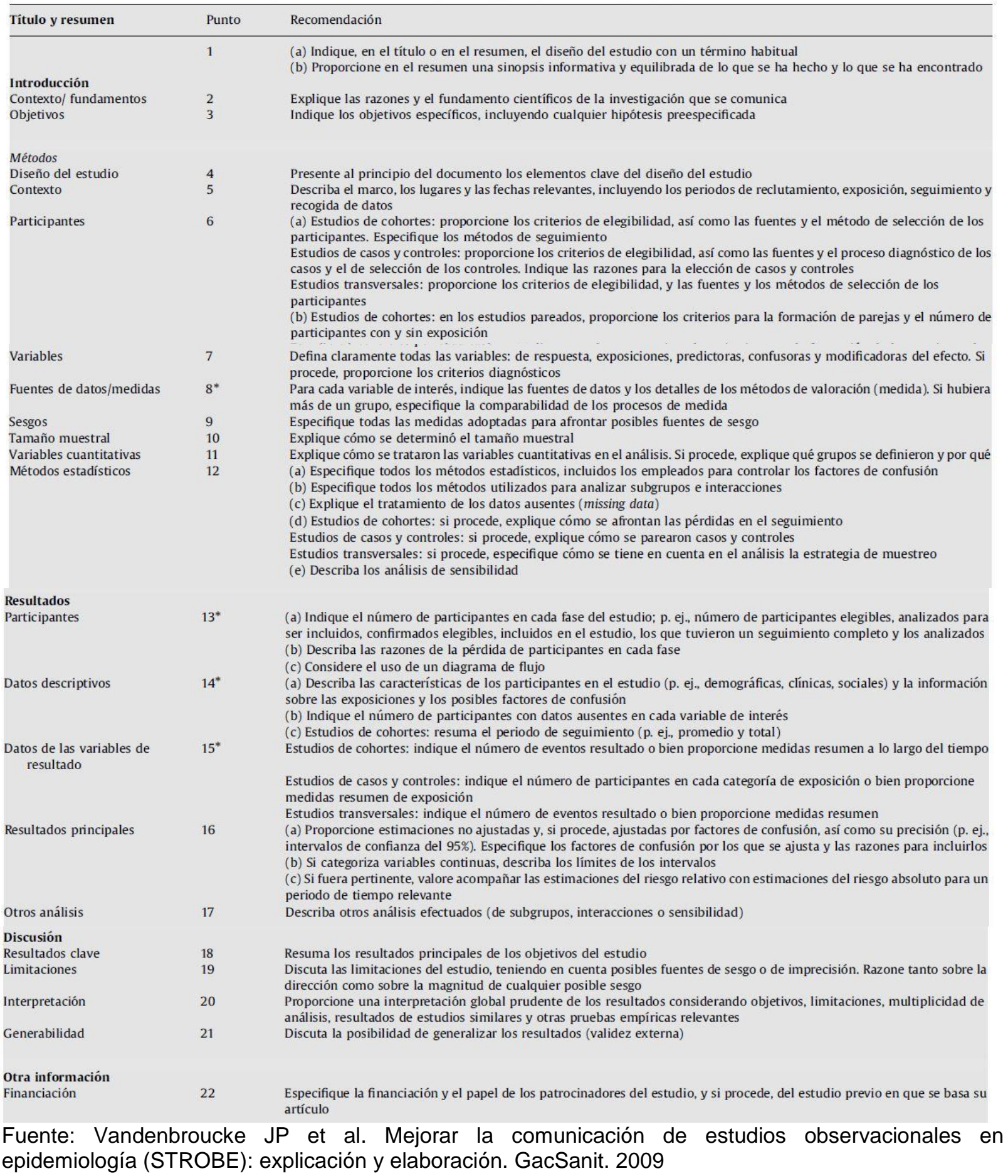




\section{Anexo II: Declaración PRISMA}

Tabla 1

Lista de comprobación de los ítems para incluir en la publicación de una revisión sistemática (con o sin metaanálisis). La declaración PRISMA

\begin{tabular}{|c|c|c|}
\hline Sección/tema & Número & Ítem \\
\hline \multicolumn{3}{|l|}{ Titulo } \\
\hline Título & 1 & Identificar la publicación como revisión sistemática, metaanálisis o ambos \\
\hline \multicolumn{3}{|r|}{ 然 } \\
\hline Resumen estructurado & 2 & $\begin{array}{l}\text { Facilitar un resumen estructurado que incluya, según corresponda: antecedentes; objetivos; } \\
\text { fuente de los datos; criterios de elegibilidad de los estudios, participantes e intervenciones; } \\
\text { evaluación de los estudios y métodos de síntesis; resultados; limitaciones; conclusiones e } \\
\text { implicaciones de los hallazgos principales; número de registro de la revisión sistemática }\end{array}$ \\
\hline \multicolumn{3}{|l|}{ Introducción } \\
\hline Justificación & 3 & Describir la justificación de la revisión en el contexto de lo que ya se conoce sobre el tema \\
\hline Objetivos & 4 & $\begin{array}{l}\text { Plantear de forma explícita las preguntas que se desea contestar en relación con los } \\
\text { participantes, las intervenciones, las comparaciones, los resultados y el diseño de los estudios } \\
\text { (PICOS)* }\end{array}$ \\
\hline \multicolumn{3}{|l|}{ Métodos } \\
\hline Protocolo y registro & 5 & $\begin{array}{l}\text { Indicar si existe un protocolo de revisión al que se pueda acceder (por ej., dirección web) y, si } \\
\text { está disponible, la información sobre el registro, incluyendo su número de registro }\end{array}$ \\
\hline Criterios de elegibilidad & 6 & $\begin{array}{l}\text { Especificar las características de los estudios (por ej., PICOS, duración del seguimiento) y de las } \\
\text { características (por ej., años abarcados, idiomas o estatus de publicación) utilizadas como } \\
\text { criterios de elegibilidad y su justificación }\end{array}$ \\
\hline Fuentes de información & 7 & $\begin{array}{l}\text { Describir todas las fuentes de información (por ej., bases de datos y períodos de búsqueda, } \\
\text { contacto con los autores para identificar estudios adicionales, etc.) en la búsqueda y la fecha } \\
\text { de la última búsqueda realizada }\end{array}$ \\
\hline Búsqueda & 8 & $\begin{array}{l}\text { Presentar la estrategia completa de búsqueda electrónica en, al menos, una base de datos, } \\
\text { incluyendo los límites utilizados, de tal forma que pueda ser reproducible }\end{array}$ \\
\hline
\end{tabular}

Fuente: Urrútia G et al. Declaración PRISMA: una propuesta para mejorar la publicación de revisiones sistemáticas y metaanálisis. Med Clin (Barc). 2010; 135(11): 507-

Recibido: 18 de febrero 2016;

Aceptado: 14 de mayo 2016

ISSN 1695-6141 